\title{
Conducta diurna del chungungo Lontra felina (Molina, 1782) en dos localidades de la costa de Talcahuano, Chile: ¿efectos de la exposición al oleaje y de las actividades humanas?
}

\author{
Daylight behavior of the sea otter Lontra felina (Molina, 1782) in two localities off the coast of \\ Talcahuano, Chile: effects of wave exposure and human activities? \\ Manuel Badilla' ${ }^{1}$ y Mario George-Nascimento ${ }^{2}$ \\ ${ }^{1}$ OBC Chinchimen, Avda del Mar N $N^{a} 3072$ - Maitencillo, Chile \\ ${ }^{2}$ Departamento de Ecología Costera, Facultad de Ciencias, Universidad Católica de la Santísima Concepción \\ Casilla 297, Concepción, Chile \\ mgeorgen@ucsc.cl
}

\begin{abstract}
The biology of the South American sea otter (Lontra felina) is scarcely known along the Chilean coast. In this study, habitat use during day time, and the behavior of this species are described, and compared between two localities: a protected embayment inhabited by artisanal fishermen and, an area of difficult access, both on the central-southern coast of Chile. The study was carried out at the end of 2005 and beginning of 2006. Despite the vicinity of the two studied localities (separated by ca. $9 \mathrm{~km}$ ), there were differences in the time of peak diurnal activities, in duration of diving, and in the proportion of time devoted to socialization. At the most isolated and exposed locality sea otters were seen more frequently transiting in the water, and making long dives, while at the protected site, in vicinity of the fishermen village, diving was more frequent and shorter. Habitat use was also different between localities according to the presence of human activities. Some of these results contrast with other reports from Chile and highlight the need to improve the knowledge of the factors affecting the behavior of Lontra felina.
\end{abstract}

Keywords: Behavioral patterns, antropic presence, local variability

\section{Introducción}

Lontra felina (Molina, 1782) (Carnivora: Mustelidae) es la especie de nutria más pequeña de su género y la única cuya distribución neotropical es exclusivamente marina (Ostfeld et al. 1989). Se encuentra desde Perú (6º S) hasta el Cabo de Hornos (56S) (Castilla \& Bahamondes 1979, Ebensperger \& Castilla 1992, Medina-Vogel et al. 2004). El hábitat más utilizado por L. felina es el litoral rocoso escarpado y expuesto, con presencia de galerías naturales, y su actividad se desarrolla en un ámbito de hogar que abarca cerca de $4 \mathrm{~km}$ lineales de costa y $100 \mathrm{~m}$ mar adentro, hasta $30 \mathrm{~m}$ al interior de la franja costera continental (Castilla \& Bahamondes 1979, Cabello 1983, Sielfeld 1990, Ebensperger \& Castilla 1992, Medina-
Resumen.- La biología del chungungo (Lontra felina) en la costa de Chile es poco conocida. En este estudio se describe el uso diurno del hábitat y las conductas que realiza esta especie, y se las compara entre una caleta de pescadores y otro lugar de difícil acceso en el sur de Chile. El estudio se realizó a fines del 2005 y principio del 2006. Pese a la cercanía de las localidades estudiadas (cerca de $9 \mathrm{~km}$ ), se observaron diferencias en los máximos de actividad diurna, en la extensión de los buceos y en la proporción del tiempo dedicado a socialización. En la localidad más expuesta al oleaje y de más difícil acceso fue más frecuente observar al chungungo en tránsito, y efectuando buceos más extensos. En la localidad más protegida fue más frecuente observarlos buceando, interactuando con otras especies y socializando. El uso del hábitat en presencia y ausencia de actividades humanas también arrojó diferencias entre localidades. Algunos de estos resultados se contraponen a hallazgos en otras partes de Chile y resaltan la necesidad de mejorar el conocimiento acerca de los factores que afectan la conducta de Lontra felina.

Palabras clave: Patrones conductuales, presencia antrópica, variabilidad local

Vogel et al. 2004, 2006). Las causas principales de la disminución poblacional y distribución fragmentada del chungungo en Chile son la caza ilegal con fines peleteros (alrededor de 38.000 individuos entre 1910-1954), el conflicto con la pesquería, y la destrucción e invasión de su hábitat, por lo que estaría al borde de la extinción en diversas localidades (Castilla \& Bahamondes 1979, Cabello 1983, Glade 1993, CONAMA 2007). Como consecuencia de esto, su estado de conservación es el de una especie en peligro (IUCN 2008).

La escasa información disponible sobre su ecología, fisiología y conducta hacen difícil la formulación de planes de manejo enfocados a su conservación (Cabello 1983, Soto 1996). Sin embargo, los estudios recientes 
señalan la importancia de la disponibilidad de alimento y del grado de exposición al oleaje como factores determinantes de, por ejemplo, la extensión de los buceos de L. felina (Villegas et al. 2007). Además, se ha demostrado el efecto directo e indirecto de las actividades humanas en la conducta de L. felina en un amplio rango geográfico de Chile central (Medina-Vogel et al. 2007, 2008).

El marco conceptual de la teoría de forrajeo óptimo (Stephen \& Krebs 1986) ha sido empleado en estudios en esta especie para explicar las modificaciones de su conducta de alimentación en ambientes expuestos y protegidos (Villegas et al. 2007). Sin embargo, en presencia de depredadores o amenazas equivalentes, como pueden ser algunas actividades humanas, los organismos podrían hacer uso de hábitats subóptimos (Townsend 2003). Otro cuerpo de ideas aplicado al estudio de los patrones de actividad diaria de Lontra felina, ahora basado en la ecofisiología, señala a la latitud como un factor determinante, ya que en la zona centro norte de Chile se ha registrado una mayor actividad del chungungo en horas crepusculares (Castilla \& Bahamondes 1979), en contraste a dos máximos de actividad diurna en el sur de Chile (Sielfeld \& Castilla 1999).

Este estudio tiene por objeto comparar la conducta y el uso del hábitat Lontra felina entre dos localidades costeras de la región del Biobío, en la costa centro-sur de Chile, que contrastan en el grado de exposición al oleaje (Villegas et al. 2007) y la presencia de actividades humanas (Medina-Vogel et al. 2008), ambas señaladas como determinantes de las variaciones conductuales de esta especie.

\section{Material y métodos}

Entre octubre de 2005 y enero de 2006 se realizaron 10 sesiones de observación sobre la conducta de Lontra felina, en dos localidades costeras de la región del Biobío, Chile: caleta Chome $(n=6)$ y en Punta Lobos $(n=4)$, consideradas como focos regionales de alta biodiversidad (Fig. 1, CONAMA 1999). Una de las localidades, Chome, está ubicada en el extremo sur de la península Hualpén (3646’25"S y 7312’42"W), y se caracteriza por ser una zona rocosa protegida donde se desarrollan frecuentes actividades de pesca artesanal, buceo y extracción de mariscos. Observaciones previas permiten estimar un máximo de cuatro ejemplares en este sitio. La otra localidad, Punta Lobos, está en el extremo sur de la península Tumbes (36²'40"S; 7309’41"W), y se caracteriza por poseer una costa rocosa escarpada expuesta, formada por acantilados de difícil acceso en donde se observó un máximo de tres individuos. Esta última es la localidad con menor presencia de humanos. Ambas localidades están separadas linealmente por cerca de 9 km (Fig. 1).

La metodología empleada en este estudio está basada en el avistamiento de ejemplares de Lontra felina. Sin embargo, cabe reconocer que ésta posee limitaciones ya que sólo se puede registrar la actividad durante el día, lo que puede ser superado al emplear radio-telemetría, permitiendo así un registro continuo e independiente de la disponibilidad de luz solar (Medina-Vogel et al. 2007). En este estudio, una sesión de observación es un día de trabajo en terreno, de 10:00 a 19:00 h, y puede constar de un máximo de 9 eventos de observación, cuya duración fue de $50 \mathrm{~min}$ (con $10 \mathrm{~min}$ de descanso). Sin embargo, no fue posible realizar todos los eventos principalmente por condiciones climáticas adversas. Las observaciones se realizaron en tres rangos horarios (10:00-13:00 h, 13:00-16:00 h y 16:00-19:00 h).

Las observaciones fueron realizadas con binoculares 8 x 30 desde lugares seleccionados para minimizar el que los animales detectaran la presencia del observador. La duración de las diferentes conductas fue cronometrada en minutos. Se consideró como avistamiento la individualización visual de un chungungo. En las observaciones se distinguieron las siguientes conductas (sensu Martin \& Bateson 1993): a) alimentación, b) descanso, c) tránsito (nado en superficie, desplazamiento en tierra), d) socialización, e) buceo, f) acicalamiento. En relación al uso del hábitat se consideraron las siguientes dos categorías: a) avistamiento en el agua, b) avistamiento en sustrato sólido (roca, botes, boyas, etc). También se registró la duración de cada conducta, hora del día, y si había humanos que los animales pudiesen detectar (aproximadamente a no más de $60 \mathrm{~m}$, en forma independiente de su cantidad y proximidad). Sin embargo, no se consideró ni la distancia, cantidad o duración de la presencia de humanos en los análisis estadísticos.

En cada localidad se elaboraron mapas de distribución de los avistamientos, y las observaciones fueron clasificadas según presencia o ausencia de actividades humanas para cada localidad, y según una línea aproximadamente 'paralela', a $50 \mathrm{~m}$ de la costa y perpendicular a la visión del observador, ya que Lontra felina usa principalmente la franja de $100 \mathrm{~m}$ desde la costa (Castilla \& Bahamondes 1979, Cabello 1983, Sielfeld 1990, Medina-Vogel et al. 2007, 2008). Esta línea divide los avistamientos como: lejos, para aquellos que se encuentran más allá de la línea de los 50 m, y cerca, los restantes. 


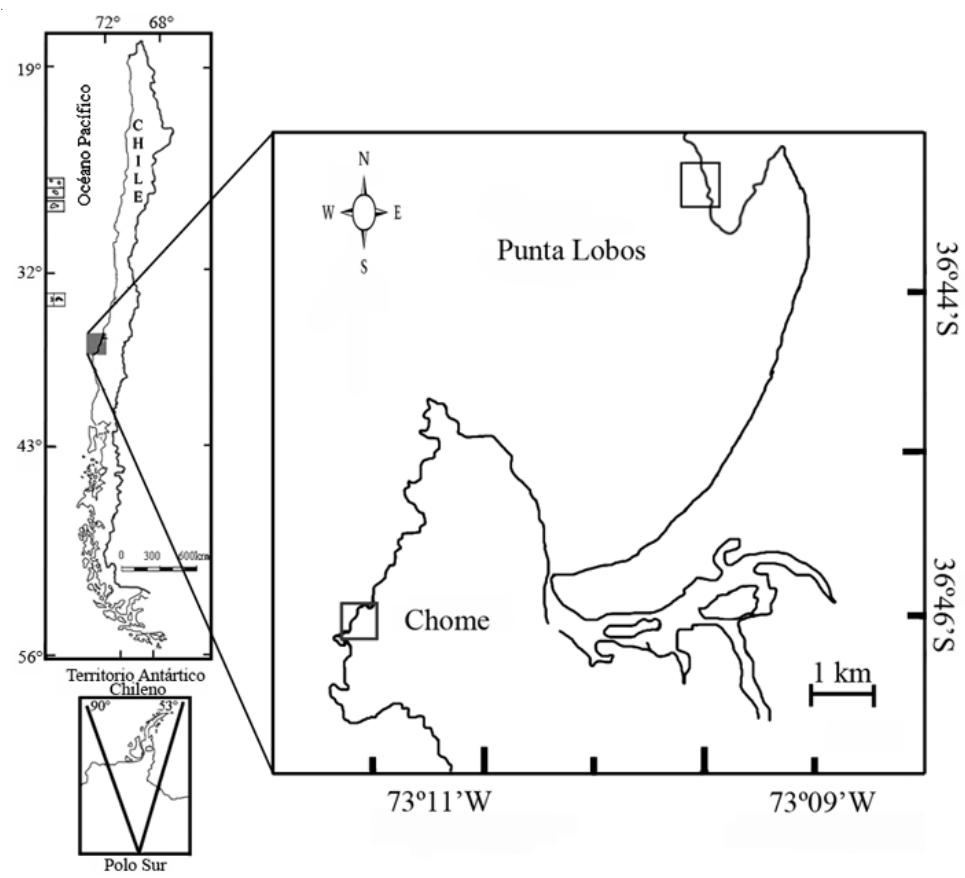

Figura 1

Ubicación geográfica de las dos localidades de la costa de Talcahuano, Chile, donde se estudió la conducta de Lontra felina

Geographic location of two sites off the coast of Talcahuano, Chile, where the behavior of Lontra felina was studied

Las frecuencias de avistamiento según hábitat, localidad y tipo de conducta se analizaron por medio de tablas de contingencia y su significancia estadística fue evaluada con pruebas de Chi-cuadrado (Zar 1996). En forma similar se examinaron las frecuencias de avistamiento en ambas localidades según su cercanía a la costa y a la presencia de actividades humanas. La significancia estadística de la diferencia entre localidades en la extensión de los buceos de Lontra felina fue evaluada mediante la prueba de Wilcoxon-Mann-Whitney para dos muestras (Zar 1996).

Este estudio no incluyó observaciones subacuáticas, por lo cual se desconoce la profundidad adyacente a los sitios de estudio y el tipo de fondo (roca, arena, algas u otro). Esto es un factor importante al estudiar la actividad del chungungo, por su efecto sobre la diversidad de alimentos o su escasez, y por consiguiente, por su efecto sobre la frecuencia de los buceos.

\section{Resultados}

En total, se llevaron a cabo 23 eventos de observación con 296 avistamientos y 275,4 min de actividad en Chome, y 22 eventos de observación con 144 avistamientos y 347,2 min de actividad en Punta Lobos
(Tablas 1 y 2). Las frecuencias de avistamiento según rangos horarios muestran diferencias significativas entre las localidades $\left(\chi^{2}=21,8\right.$; g.l. $\left.=2 ; P<0,001\right)$. El máximo de actividad diurna observado en Chome se encuentra entre las 10:00 y 13:00 h, con el 56,0\% de los avistamientos (Fig. 2), y el 60,2\% del tiempo cronometrado en actividad (Fig. 3). En Punta Lobos el patrón de actividad mostró un máximo entre las 13:00 y 16:00 h, con el 68,8\% de los avistamientos (Fig. 2) y un 66,7 \% del tiempo cronometrado en actividad (Fig. 3).

El hábitat más utilizado en ambas localidades fue el agua (Chome: 90,1\% de los avistamientos y 64,7\% del tiempo cronometrado; Punta Lobos: $81,5 \%$ de los avistamientos, y 55,4\% del tiempo cronometrado, Tablas 1 y 2). Sin embargo, la proporción de avistamientos de Lontra felina en el hábitat roca en Punta Lobos fue mayor que en Chome ( $\chi^{2}=11,4$; g.l. $=1 ; P<0,001$, Tabla 1$)$.

La frecuencia con que se observaron distintos tipos de conducta mostró diferencias significativas entre las localidades ya que fue más frecuente observar a Lontra felina alimentándose y acicalándose en Chome, y transitando en Punta Lobos $\left(\chi^{2}=44,0\right.$; g.l. $\left.=5 ; P<0,001\right)$. Otras diferencias entre localidades son que en Chome fue significativamente más frecuente ver a $L$. felina 
Tabla 1

Número de avistamientos de Lontra felina en dos localidades costeras vecinas a Talcahuano, Chile, según seis categorías de conducta y dos tipos de hábitat

Number of sightings of Lontra felina at two coastal localities near Talcahuano, Chile, according to six behavioral categories and two habitat types

\begin{tabular}{lrrrrr}
\hline $\begin{array}{c}\text { Categoría } \\
\text { conductual }\end{array}$ & \multicolumn{2}{c}{ Chome } & \multicolumn{3}{c}{ Punta Lobos } \\
\hline & Agua & Roca & Agua & Roca & Total \\
Alimentación & 51 & 5 & 3 & 12 & 71 \\
Tránsito & 56 & 0 & 66 & 3 & 125 \\
Buceo & 130 & - & 48 & - & 178 \\
Descanso & 12 & 0 & 0 & 3 & 15 \\
Acicalamiento & 0 & 15 & 0 & 6 & 21 \\
Socialización & 25 & 2 & 0 & 3 & 30 \\
Total & 274 & 22 & 117 & 27 & 440 \\
\hline
\end{tabular}

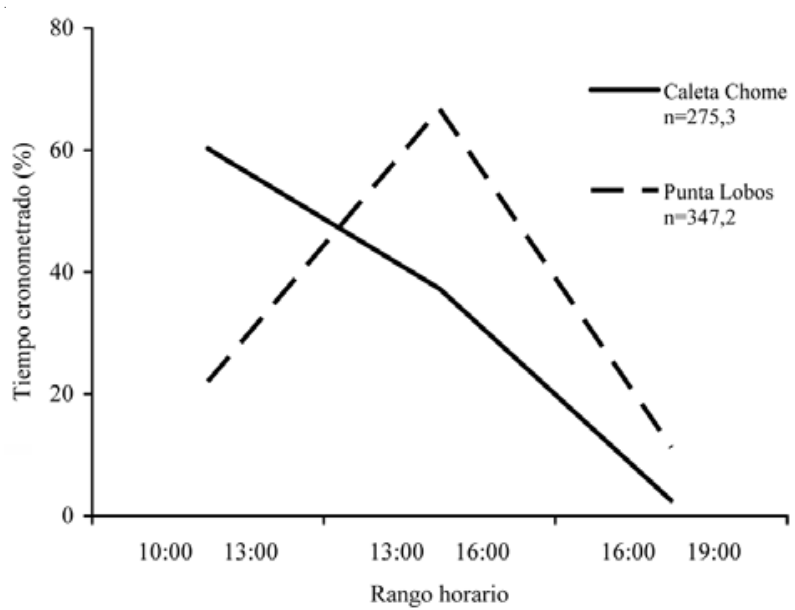

Figura 2

Porcentaje del total de tiempo cronometrado (en min) que Lontra felina ocupó en actividades diurnas en dos localidades costeras de Chile centro-sur, según tres rangos horarios

Percent of total measured time (in min), spent by Lontra felina in diurnal activities at two sites off central-south Chile, according to three time intervals
Tabla 2

Duración (minutos) de seis categorías de conducta observadas en Lontra felina, en dos tipos de hábitat, en dos localidades costeras vecinas a Talcahuano, Chile

Duration (minutes) of six behavioral categories observed in Lontra felina in two types of habitat, at two coastal localities near Talcahuano, Chile

\begin{tabular}{lrrrrr}
\hline $\begin{array}{c}\text { Categoría } \\
\text { conductual }\end{array}$ & \multicolumn{2}{c}{ Chome } & \multicolumn{3}{c}{ Punta Lobos } \\
\hline & Agua & Roca & Agua & Roca & Total \\
& 27,5 & 22,5 & 1,0 & 74,1 & 125,1 \\
Alimentación & 57,5 & 0,0 & 143,2 & 1,2 & 201,9 \\
Tránsito & 77,3 & - & 48,0 & - & 125,3 \\
Buceo & 3,0 & 0,0 & 0,0 & 4,0 & 7,0 \\
Descanso & 0,0 & 63,1 & 0,0 & 45,4 & 108,5 \\
Acicalamiento & 21,6 & 2,8 & 0,0 & 30,3 & 51,7 \\
Socialización & 186,9 & 88,4 & 192,2 & 155,0 & 622,5 \\
Total & & & & &
\end{tabular}

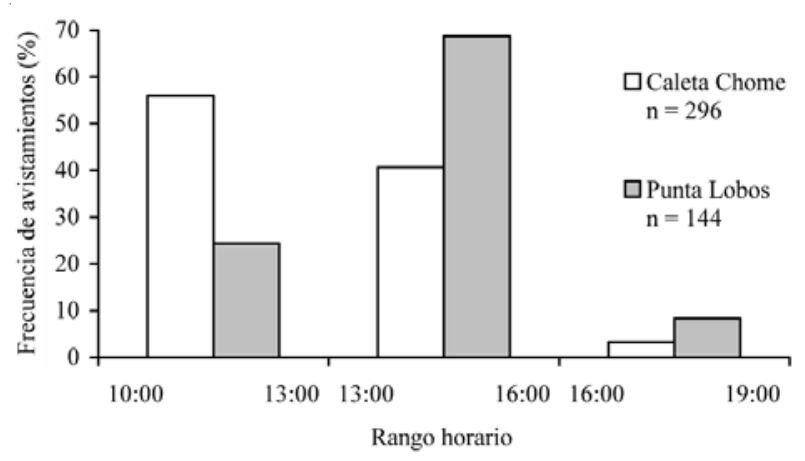

Figura 3

Frecuencia de avistamientos, en porcentaje respecto al total de avistamientos, registrados para Lontra felina costeras del centro-sur de Chile, según tres rangos horarios

Frequency of sightings, as percentage of total number of sightings, recorded for Lontra felina at two coastal sites off central-south Chile, according to three time intervals 


\section{Tabla 3}

\section{Número de avistamientos de Lontra felina según categorías de cercanía a la costa, y presencia (P) o ausencia (A) de actividades humanas en dos localidades cercanas a Talcahuano, Chile}

Number of sightings of Lontra felina according to categories of distance from the coast, and the presence $(\mathrm{P})$ or absence $(\mathrm{A})$ of human activities at two localities off the coast of Talcahuano, Chile

\begin{tabular}{lrrccr}
\hline & \multicolumn{6}{c}{ Chome } & \multicolumn{4}{c}{ Punta Lobos } & Total \\
& P & A & P & A & \\
& & & & & \\
\hline Cerca de la costa & 158 & 133 & 17 & 56 & 364 \\
Lejos de la costa & 0 & 5 & 35 & 36 & 76 \\
Total & 158 & 138 & 52 & 92 & 440 \\
\hline
\end{tabular}

alimentándose en el agua $\left(\chi^{2}=29,0\right.$; g.l. $\left.=1 ; P<0,0001\right)$ y socializando en el agua (Prueba de la probabilidad exacta de Fisher, $P=0,0025$, Tabla 1$)$. La conducta más frecuente en Chome fue el buceo, con el 43,3\% de los avistamientos, y el 26,7\% del tiempo cronometrado. De 130 avistamientos con actividad de buceo que fueron cronometrados en esta localidad, 8 mostraron a los animales con presas, y la duración promedio fue de 36,0 + 18,25 s. En tanto, en Punta Lobos, la conducta más frecuente fue el tránsito, con el 47,9\% de los avistamientos, y el 41,6\% del tiempo total cronometrado. En esta localidad se registraron 48 avistamientos en actividad de buceo con una duración promedio de $60+23,30 \mathrm{~s}$, en los que se observó que en sólo dos oportunidades manipulaban presas. La extensión de los buceos fue significativamente mayor en Punta Lobos (Prueba de dos muestras de Wilcoxon, $\mathrm{U}=1,368, P$ $<0,0001)$.

En Chome fue más frecuente avistar chungungos cerca de la costa que en Punta Lobos $\left(\chi^{2}=150,4\right.$; g.l. $=1$; $P<$ 0,0001, Tabla 3, Fig. 4). Sin embargo, en Punta Lobos hubo una mayor proporción de avistamientos cerca de la costa cuando los humanos no estaban presentes (56 de 92), que cuando estaban presentes (17 de 52, $\chi^{2}=9$,46; g.l. = $1 ; P<0,01$, Tabla 3, Fig. 4). En ausencia de actividades humanas, se registraron más avistamientos cerca de la costa en Chome (133 de 138) que en Punta Lobos (56 de 92, $\chi^{2}=45,17$; g.l. $=1 ; P<0,0001$, Tabla 3, Figura 4).

\section{Discusión}

Los resultados divergen parcialmente de lo observado en otros estudios en Lontra felina en Chile, ya que, entre otras cosas, indican sólo un máximo de actividad diurna por localidad y a diferentes horas del día (Figs. 2 y 3), que además se diferenciaron entre las dos localidades consideradas en este estudio. Además, L. felina efectuó buceos más largos en la localidad más expuesta, en contraste con lo registrado en isla Choros, en el norte de Chile (Villegas et al. 2007), donde se interpretó que la diferencia en tiempos de buceo entre dos localidades de una misma isla se podrían deber al grado de exposición al oleaje, y a la disponibilidad de presas. En este caso, muchos de los factores que han sido señalados como relevantes para entender las variaciones conductuales de L. felina no fueron considerados en este estudio (tipo y disponibilidad de hábitats, presas, refugios, ver Villegas et al. 2007). En consecuencia, no podemos concluir si lo observado se debe o no a diferencias en productividad entre las localidades.

Sin embargo, algunas observaciones se ajustan a lo esperado según el tipo de ambiente. Por ejemplo, Lontra felina mostró que consumía sus presas más frecuentemente sobre las rocas en Punta Lobos, donde sería más difícil maniobrar en el agua. En contraste, en la localidad con mayor protección del embate del oleaje (Chome), se observó una mayor frecuencia de descanso, socialización y consumo de presas en el agua (Tablas 1 y 2). Aunque en nuestros datos L. felina fue avistada principalmente en el agua (Tablas 1 y 2), esta especie pasaría cerca del 80\% de su tiempo en tierra (Medina-Vogel et al. 2007). Esta diferencia con el estudio recién mencionado es posible que obedezca a que en él se empleó radiotelemetría, lo que permite un registro continuo de su actividad, aunque el animal no esté visible. En contraste, en este estudio la conducta se evaluó en base a avistamientos, lo que hace más fácil ver al chungungo mientras está en el agua durante el día.

Otros factores que no han sido considerados al momento de evaluar su conducta permitirían explicar de las diferencias encontradas entre los estudios en los porcentajes del tiempo dedicados a distintas conductas, que podrían estar asociadas a las distintas épocas del año, o a la presencia de crías en las madrigueras, o a la presencia de competidores. Al respecto, y pese a que la conducta de tránsito se observó con similar frecuencia en ambas localidades, el chungungo le destinó más tiempo en Chome (Tablas 1 y 2). Esto puede deberse en parte a que en esta localidad se observaron adultos acompañados de crías varios meses después de terminadas las observaciones hechas (febrero-abril 2006). Es decir que en el período en que fueron hechos los registros en Chome habría habido crías en etapa de desarrollo en las madrigueras, por lo que se podría entender el alto porcentaje de tiempo en conductas de tránsito y buceo, y 
A) Chome
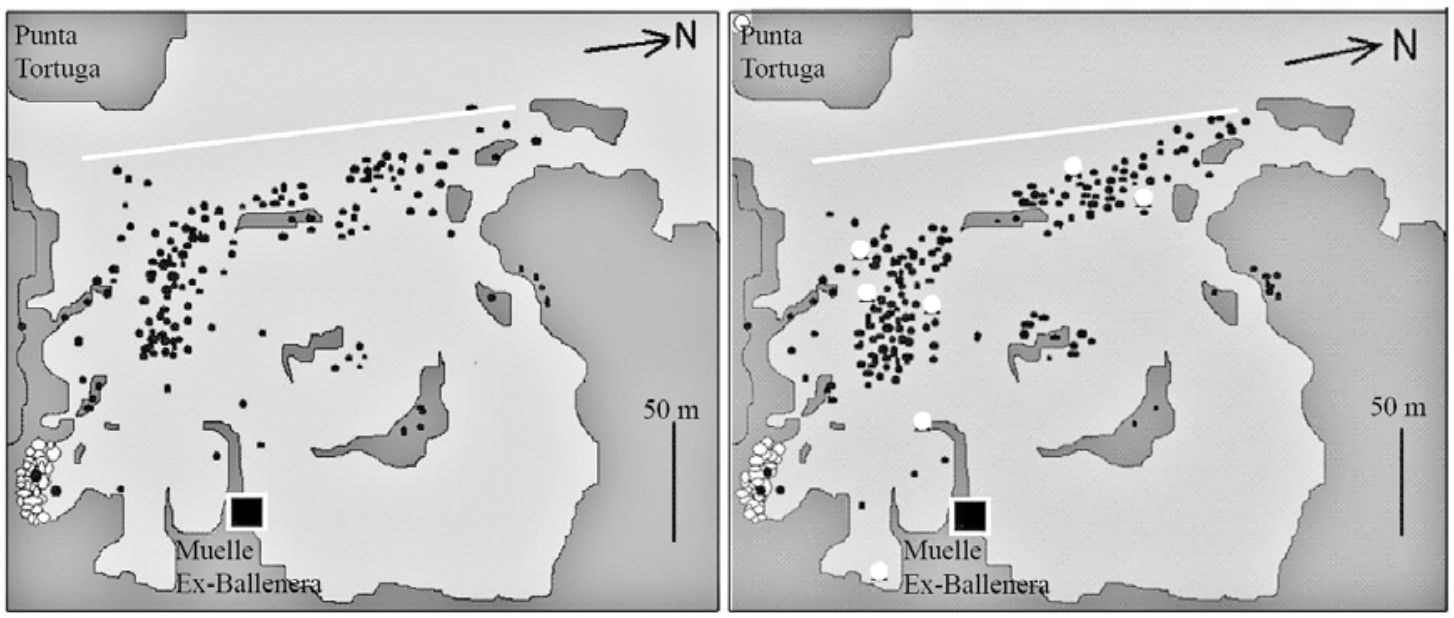

B) Punta de Lobos
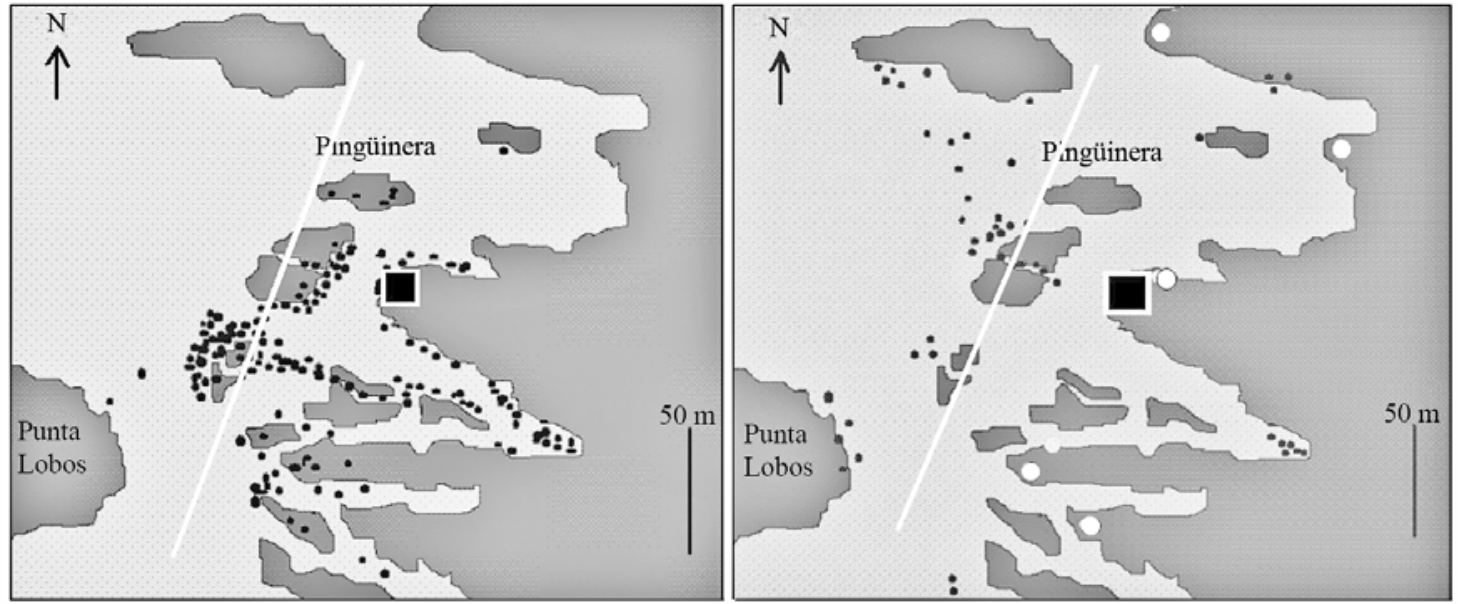

Figura 4

Diagrama de los avistamientos (puntos negros) de Lontra felina en a) Chome, y b) Punta Lobos, según ausencia (izquierda) o presencia de actividades humanas (derecha). El cuadrado negro indica el lugar del observador, los círculos blancos indican actividad humana. La línea blanca divide el espacio en dos sectores: cerca y lejos de la costa

Diagram of sightings of Lontra felina (black dots) at a) Chome and b) Punta Lobos, according to absence (left) or presence (right) of human activities. Black square = observer position, white circles $=$ human activities. The white line divides the space into two areas: near or far from the shoreline

los constantes regresos a la madriguera con presas a medio consumir, destinando de esta forma gran cantidad de tiempo para cacería. En Punta Lobos en tanto, la mayoría de las conductas registradas en el agua fueron de tránsito (Tabla 1), con una baja frecuencia de buceos (Tabla 2), casi el doble más extensos que en Chome, y con una alta frecuencia de consumo de presas en las rocas. Esto último podría asociarse a que sólo en Chome se observó que especies como las gaviotas (Larus dominicanus) y cormoranes (Phalacrocorax brasilianus) los acosan mientras se alimentan en el agua, aunque esto no fue cuantificado.

El efecto de las actividades humanas sobre la conducta de Lontra felina tiene una doble faceta (MedinaVogel et al. 2007, 2008). Cuando los humanos son hostiles (o sus mascotas), esta especie ve amenazada su 
supervivencia, en tanto que cuando los humanos resultan en aportes de desperdicios que les sirven de alimento se transforman en un factor positivo. La conducta oportunista y despreocupada del chungungo observada en Chome es de larga data, según los lugareños, llegando a arrebatar la pesca sobre botes y a los propios buzos, lo que estaría asociado a la presencia de humanos no hostiles. Además, es común observar conductas de alimentación, descanso, acicalamiento o alimentación en la superficie del agua, botes o rocas. Estos antecedentes pueden ayudar a explicar la predominancia de actividades en el agua en esta localidad, una alta frecuencia de buceos cortos, y en su mayoría cerca de la costa, e independientemente de la presencia de actividades humanas (Tabla 3, Fig. 4), lo que se ha descrito en otras especies del género Lontra, y en general entre los mustélidos (Sielfeld \& Castilla 1999, Ottino \& Giller 2004, Polednik et al. 2004). En contraste, en Punta Lobos, el chungungo evita a los seres humanos, lo que explicaría su permanencia lejos de la madriguera gran parte del día, favoreciendo así el encuentro de grietas de difícil acceso y escasa visibilidad desde la costa. En suma, más que la presencia de actividades antrópicas, una posible respuesta a la diferencias conductuales observadas entre chungungos de ambas localidades podría ser la forma en que se realizan éstas. Si bien en ambos sitios se desarrollan actividades humanas, la hostilidad con la que se realizan en Punta Lobos parece ser determinante para el desenvolvimiento diario del chungungo en áreas alejadas de su madriguera.

\section{Literatura citada}

Cabello C. 1983. La nutria de mar en la isla de Chiloé. Boletín Técnico, CONAF 6: 1-37.

Castilla JC \& I Bahamondes. 1979. Observaciones conductuales y ecológicas sobre Lutra felina (Molina, 1782) (Carnivora, Mustelidae) en la zona central y centro-norte de Chile. Archivos de Biología y Medicina Experimentales 12: 119-132.

CONAMA. 1999. Talleres de gestión ambiental local para líderes comunitarios y juveniles de la comunidad de Talcahuano, 111 pp. CIDEM Consultores SA, Concepción.

CONAMA. 2007. Reglamento para clasificación de especies silvestres. [en línea] <http://www.conama.cl/ clasificacionespecies/index.htm>

Ebensperger LA \& JC Castilla. 1992. Selección de hábitat en tierra por nutria marina, Lutra felina, en Isla Pan de Azúcar, Chile. Revista Chilena de Historia Natural 65: 429-434.

Glade A. 1993. Libro rojo de los vertebrados terrestres de Chile, 67 pp. Corporación Nacional Forestal, Santiago.

IUCN. 2008. IUCN Red List of Threatened Species. [en línea] $<$ http//www.redlist.org $>$
Martin P \& P Bateson. 1993. Measuring behaviour: an introductory guide, 242 pp. Cambridge University Press, Cambridge.

Medina-Vogel G, CR Delgado, RE Alvarez \& JLV Bartheld. 2004. Feeding ecology of the marine otter (Lutra felina) in a rocky seashore of the south of Chile. Marine Mammal Science 20: 134-144.

Medina-Vogel G, JLV Bartheld, RE Alvarez \& CR Delgado. 2006. Population assessment and habitat use by marine otter (Lontra felina) in southern Chile. Wildlife Biology 12: 191-199.

Medina-Vogel G, L Merino, R Monsalve-Alarcón \& J de A Vianna. 2008. Coastal marine discontinuities, critical patch size and isolation: implications for marine otter conservation. Animal Conservation 11: 57-64.

Medina-Vogel G, F Boher, G Flores, A Santibáñez \& C Soto-Azat. 2007. Spacing behavior of marine otters (Lontra felina) in relation to land refuges and fishery waste in central Chile. Journal of Mammalogy 88: 487-494.

Ostfeld RS, L Ebensperger, LL Klosterman \& JC Castilla. 1989. Foraging, activity budget and social behaviour of the South American marine otter Lutra felina (Molina, 1782). National Geographic Research 5: 422-438.

Ottino P \& PS Giller. 2004. Distribution, density, diet and habitat use of the otter in relation to land use in the Araglin Valley, Southern Ireland. Biology and Environment: Proceedings of the Royal Irish Academy 104B: 1-17.

Polednik L, R Mitrenga, K Polednikova \& B Lojkasek. 2004. The impact of methods of fishery management on the diet of otters (Lutra lutra). Folia Zoologica 53: 27-36.

Sielfeld W. 1990. Características del hábitat de Lutra felina (Molina) y L. provocax Thomas (Carnivora, Mustelidae) en Fuego-Patagonia. Revista de Investigaciones Científicas y Tecnológicas, Serie Ciencias del Mar 1: 30-36.

Sielfeld W \& JC Castilla. 1999. Estado de conservación y conocimiento de las nutrias en Chile. Estudios Oceanológicos 18: 69-79.

Soto R. 1996. Estructura gremial de un ensamble de depredadores de la zona intermareal rocosa en Chile central. Investigaciones Marinas 24: 97-105.

Stephens DW \& JR Krebs. 1986. Foraging theory, 247 pp. Princeton University Press, Princeton.

Townsend CR. 2003. Individual, population community and ecosystem consequences of a fish invader in New Zealand streams. Conservation Biology 17: 38-47.

Villegas MJ, AAron \& LA Ebensperger. 2007. The influence of wave exposure on the foraging activity of marine otter, Lontra felina (Molina, 1782) (Carnivora: Mustelidae) in northern Chile. Journal of Ethology 25: 281-286.

Zar JH. 1996. Biostatistical analysis, 662 pp. Prentice Hall, Englewood Cliffs. 